\title{
Do Older T'ai Chi Practitioners Have Better Attention and Memory Function?
}

\author{
David W.K. Man, PhD, ${ }^{1}$ William W.N. Tsang, PhD, ${ }^{1}$ and Christina W.Y. Hui-Chan, $\mathrm{PhD}{ }^{2}$
}

\begin{abstract}
Objectives: Cognitive declines are common in older people and can be a major health issue in an aging world. One type of body-mind exercises, $t^{\prime} a i$ chi, can be a possible means to help maintaining older adults' cognitive abilities, in addition to beneficial effects of physical exercises. The purpose of this study was to investigate whether t'ai chi practitioners had better attention and memory functions than older people with or without regular exercises.

Methods: A cross-sectional study examining the relationship between t'ai chi practice and age-, gender- and education-similar older peoples' attention and memory functions. Forty-two (42) community-dwelling elderly subjects, aged 60 or older, recruited from t'ai chi clubs in Hong Kong formed the t'ai chi group. Another 49 elderly having regular exercise habits were recruited from community centers for inclusion in the exercise group. A nonexercise group (normal healthy control) consisting of 44 subjects were also recruited by random selection and through contacting local elderly centers. They were also screened by the Modified Barthel Index, Chinese Mini-mental Status Examination, Geriatric Depression Scale, and evaluated by attention tests (Color Trail Form A-1 and 2) and memory tests (including Rivermead Behavioral Memory Test and The Hong Kong List Learning Test).

Results: The main finding was that the three groups differed in attention and memory functions, and the $t^{\prime} a i$ chi group had demonstrated better performance than the other two groups in most subtests.

Conclusions: As a causal relationship cannot be assumed in the present cross-sectional study, future research is required to examine how t'ai chi can improve cognitive function using a randomized control trial as well as determining whether $t^{\prime}$ ai chi practice can lead to better health status among elderly people.
\end{abstract}

\section{Introduction}

$\mathrm{O}$ LDER ADULTS ARE FREQUENTLY encouraged to participate in physical exercise due to the cumulative evidence indicating physiologic benefits of exercise such as enhanced cardiovascular function, physical stamina, muscle strength, ${ }^{1,2}$ improved well-being, ${ }^{3}$ and improved neuropsychologic or cognitive performance. ${ }^{4}$ The potential for exercise-related improvements in cognitive functioning is of particular interest due to the increased prevalence of cognitive deficits among older adults. ${ }^{5-8}$ Exercise-related improvements in cognitive function have been well demonstrated. ${ }^{9}$ They are of particular interest due to cognitive aging. ${ }^{10}$

While physical exercises are popular among older adults, some of them tend to focus more on mind-body exercises such as t'ai chi, which incorporates body with movement (physical), breath, and attention training (mediation, focus- ing on event generated internally or externally) and may alleviate disease symptoms and maintain health. ${ }^{11}$ Recent research reviews have summarized various therapeutic effects of $t^{\prime}$ ai chi on older adults ${ }^{12,13}$ including cognitive status. $^{14}$

Although many researchers had reported that both physical and mind-body exercises could improve physical fitness, few have actually compared their relative beneficial effects on cognitive functions such as visualization, imagery, attention, memory, and planning. ${ }^{14,15}$ Note that $t^{\prime}$ ai chi's 108 forms are considered complex motor skill training, which demand more attention and memory throughout its practice. ${ }^{15,16}$ The need for high-level balance control and sometimes fast reaction time during t'ai chi practice also require a high degree of attention, ${ }^{17}$ which in turn could facilitate further cognitive processing such as memory. ${ }^{18}$ The relaxation effect of $t^{\prime} a i c h i^{19,20}$ may also play a part in promoting

\footnotetext{
${ }^{1}$ Department of Rehabilitation Sciences, The Hong Kong Polytechnic University, Hung Hom, Kowloon, Hong Kong.

${ }^{2}$ Department of Physical Therapy, University of Illinois, Chicago, IL.
} 
memory function. Yau and Packer's qualitative study also reported improved cognitive function, including memory, concentration span, mental alertness, and ability in problem solving. ${ }^{21}$ We therefore anticipated that repeated $t^{\prime} a i$ chi practice may enhance specific cognitive functions (attention and memory) in t'ai chi practitioners. The purpose of this study was to compare the attention and memory function among $t^{\prime}$ ai chi practitioners and older subjects similar in age, gender, and education with or without regular exercises.

\section{Methods}

\section{Study design and procedure}

This study used a cross-sectional design. Ethical approval was obtained from the Hong Kong Polytechnic University, Department of Rehabilitation Sciences Ethical Committee. Prior to assessment, informed consent was obtained individually from the $t^{\prime}$ ai chi group (TCG), exercise group (EG), and control group (CG). Independent and trained assessors administered both the screening tests and evaluation in cognitive functions for the three groups. All the assessors were blinded for the participants' group allocation.

\section{Participants}

Participants recruited for this study were aged 55 or above and included both genders. They were independent in selfcare as assessed by the Modified Barthel Index, ${ }^{22,23}$ able to communicate and follow simple verbal instructions, and demonstrated emotional stability as indicated by the Geriatric Depression Scale. ${ }^{24,25}$ Exclusion criteria included a history of systematic cardiovascular diseases, poorly controlled hypertension, stroke, Parkinson's disease, severe dizziness, cancer, cognitive problems as indicated by several instruments (see Measurement session for details) such as the Chinese version of the Mini-Mental Status Examination ${ }^{26}$ (cutoff score $=24$ out of 30), early stages of Alzheimer disease, or the use of psychoactive drugs. Elderly people pursuing regular mental activities, such as playing mahjong, chess, bridge, and reading for more than 30 minutes per session and 3 times per week, for 1 year or longer were excluded.

\section{Procedures}

Fifty (50) community-dwelling older people, aged 60 or older, were screened by staff member of $t^{\prime} a i$ chi clubs and Hong Kong social centers for the elderly according to selection criteria listed in a questionnaire, and then randomly selected from membership lists of the organizations. They then formed the TCG. They had practiced t'ai chi (Ng style) at least 3 times a week (for about 45 minutes per session) individually for 3 or more years, and were able to perform the 108 forms of $t^{\prime}$ ai chi by themselves. Among the 50 subjects, only 42 had accomplished the full evaluation process. Similarly, another 50 were screened by staff members from community centers according to different selection criteria and assigned to the exercise group (EG). They had no experience with t'ai chi, but had performed aerobic, flexibility, and stretching exercises for 3 days per week, 45 minutes per session, for at least 3 years. Thus, the frequency of exercise in the EG subjects was considered similar to that of the TCG, though not the length of practice. The exercises included morning walks, flexibility exercises and stretching, or swimming. They were repetitive and did not demand much in attention and memory once learned. Participants in sports, such as racket sports, cricket, or golf, which may involve a certain amount of attention and cognitive function, were excluded from joining the EG. Ultimately, 49 of the EG were included in the final analysis as there was one dropout due to a personal reason. A nonexercise group (normal healthy control) consisting of 50 subjects was also recruited by random selection through contacting staff members of local elderly centers. They were defined as an inactive elderly group, having no regular exercise (such as morning walk) or $t^{\prime}$ ai chi practice, but were independent in their activities of daily living, and could communicate and complete simple cognitive assessment procedures in the present study. Only 44 subjects were included in the data analysis as six of them either did not complete the assessment procedures or withdrew from the study due to health or personal reasons.

Screening tools include the following:

1. A 10-item Modified Barthel Index (MBI) with score ranging from 0 to 100 was used to conduct the routine assessment of 10 personal activities of daily living, such as personal hygiene, bathing self, feeding, and toileting. ${ }^{22,23}$ Subjects showing dependence on others would be excluded.

2. Chinese Version of Mini-Mental Status Examination (CMMSE) was a commonly used measure of global cognitive level. Subjects who scored below 24 (out of 30) were excluded from this study. ${ }^{26}$

3. Emotional stability was measured by the Geriatric Depression Scale (GDS). ${ }^{24}$ It is a screening test for depression. A shorter, translated Chinese form ${ }^{27}$ and a cutoff point of 8 or below was used in the present study to exclude those with clinically valid depressive symptoms.

\section{Primary outcome measures}

These measures were used to compare the attention and memory functions of elderly people among the TCG, EG, and CG.

1. Attention elements

Typical tests of attention such as letter cancellation and digit span (forward and backward) are not chosen in the present study as they mainly focused on sustained attention. They may not be able to test the additional attentional functions such as selective attention and mental flexibility, which are possibly related to t'ai chi practice. Thus, Color Trails Test (CTT) Form A -Trail 1 and 2 was used in the present study. ${ }^{28}$ The CTT ( 1 and 2 ) have two subtests. CTT is designed as a culture-free test and focuses on selective attention, mental flexibility, visual-spatial skills and motor speed. CTT-1 requires the individual to rapidly draw a line connecting circles numbered 1-25 in consecutive order. CTT-2 requires the respondent to rapidly draw a line between the numbered circle, maintaining the sequence of numbers, but alternating between pink and yellow colors (i.e., 1-pink, 2-yellow, 3-pink, etc.). Color trail test scoring is a reverse code measure. In other words, shorter time taken to finish the test means better performance in attention. 
2. Memory function

a. Functional, everyday memory was assessed by the Chinese version of the Rivermead Behavioral Memory Test (RBMT-CV). ${ }^{29,30}$ The RBMT is a pencil-and-paper test. It was developed to assess changes in everyday memory and comprises 12 subcomponents, testing features such as the capacity to memorize and recall a new name, recognition of previously presented unfamiliar faces and pictures of objects, recalling a brief prose passage immediately and after a delay, and the immediate and delayed recall of a simple route. ${ }^{31}$ For each subtest, two scores are produced: a simple pass/ fail or screening score, and a standardized profile score. The screening score ranges from 0 to 12 and the standardized profile score ranges from 0 to 24 . The screening score indicates a simple way of estimating whether an individual is likely to have everyday memory problems or not, while the profile score offers a more sensitive measure of change such as after intervention or over a period of time.

b. Verbal memory and memory processes were assessed by the Hong Kong List Learning Test (HKLLT). ${ }^{32}$ The HKLLT was designed to examine the following variables: (1) rate of learning (acquisition); (2) rate of forgetting (retention); (3) encoding versus retrieval deficits; (4) learning strategies including: (a) semantic clustering, (b) subjective organization ability, (c) primacy versus recency effect, and (d) concrete versus abstract concepts; (5) memory intervention including: (a) repetitive practices and (b) external organization cues; (6) intrusion er- rors; (7) preservation errors; and (8) vulnerability to interference. The HKLLT consists of a randomly presented word-list and also another word-list that is presented in blocks, with the words that are semantically related presented together. The list for the random presentation condition consisted of 16 twocharacter Chinese words, with four items from each of four categories: furniture, vegetable (both are concrete objects); relative, and country (more abstract nouns). The words were arranged randomly with the condition that no two items from the same category were presented consecutively. For the blocked condition, there were another 16 two-character words. Chinese words from four categories_clothing, flower (concrete objects); music (e.g., folk songs and opera), and occupation (more abstract nouns)-were organized into clusters based on the categories. The test consisted of three immediate recall trials, two delayed recall trials (10 and 30 minutes), and one recognition task.

\section{Results}

Descriptive statistics were conducted to examine the demographics and cognitive (attention and memory) performance of each group (TCG, EG, and CG) on each of the cognitive subtests. Analysis of variance (ANOVA) was used to compare the group difference in different cognitive test results such as CMMSE. To avoid possible type I errors due to multiple comparisons, Bonferroni correction was used so that all comparisons collectively had an $\alpha$ of 0.05 .

Table 1. Demographic Characteristics of T'ai Chi (TCG), Exercise (EG), and Control Groups (CG)

\begin{tabular}{|c|c|c|c|}
\hline \multirow[b]{2}{*}{ Characteristics } & $T C G(\mathrm{n}=42)$ & $E G(\mathrm{n}=49)$ & $C G(\mathrm{n}=44)$ \\
\hline & \multicolumn{3}{|c|}{ Number (percentage) } \\
\hline Age & $68.9(6.1,55-81)$ & $68.1(7.5,55-85)$ & $68.2(8.5,55-90)$ \\
\hline \multicolumn{4}{|l|}{ Gender } \\
\hline Male & $20(47.6 \%)$ & $23(46.9 \%)$ & $21(47.7 \%)$ \\
\hline Female & $22(52.4 \%)$ & $26(54.1 \%)$ & $23(52.3 \%)$ \\
\hline \multicolumn{4}{|l|}{ Educational level } \\
\hline Primary or below & $27(64.3 \%)$ & $34(69.4 \%)$ & $24(54.5 \%)$ \\
\hline Above primary & $15(35.7 \%)$ & $15(30.5 \%)$ & $20(45.5 \%)$ \\
\hline \multicolumn{4}{|l|}{ Working status } \\
\hline Employed & $2(4.8 \%)$ & $1(2 \%)$ & $2(4.5 \%)$ \\
\hline Retired & $40(95.2 \%)$ & $48(98 \%)$ & $42(95.5 \%)$ \\
\hline \multicolumn{4}{|l|}{ Living conditions } \\
\hline Alone & $7(16.7 \%)$ & $1(2 \%)$ & $3(6.8 \%)$ \\
\hline With family & $35(82.3 \%)$ & $48(98 \%)$ & $41(93.2 \%)$ \\
\hline \multicolumn{4}{|l|}{ Chronic illness } \\
\hline No & $12(28.6 \%)$ & $15(30.6 \%)$ & $12(27.3 \%)$ \\
\hline Yes & $30(71.4 \%)$ & $34(69.4 \%)$ & $32(72.7 \%)$ \\
\hline \multicolumn{4}{|l|}{ Medication } \\
\hline Taking medicine & $20(52.4 \%)$ & $27(55.1 \%)$ & $27(61.4 \%)$ \\
\hline \multirow[t]{2}{*}{ No need } & $22(27.6 \%)$ & $22(44.9 \%)$ & $17(38.6 \%)$ \\
\hline & \multicolumn{3}{|c|}{ Mean (SD) } \\
\hline Length of practice (years) & $7.8(4.7)$ & $11.0(12.7)$ & \\
\hline CMMSE score $(0-30)$ & $28.8(1.0)$ & $28.3(1.6)$ & $28.2(1.5)$ \\
\hline GDS score (0-15) & $6.9(3.6)$ & $6.6(5.7)$ & $6.8(4.7)$ \\
\hline
\end{tabular}

CMMSE, Chinese Version of Mini-Mental Status Examination; GDS, short form of the Geriatric Depression Scale; SD, standard deviation. 


\section{Descriptive statistics}

Table 1 shows comparisons of demographic characteristics among the three groups. No statistically significant differences were found in age, gender, educational level, CMMSE, and GDS scores across the three groups by one-way ANOVA. In other words, the groups were comparable in nature.

The social and family backgrounds and health status were also similar across the three groups. They were all fully independent in personal activities of daily living, as reflected by achieving the maximum score of 100 in the MBI.

One-way ANOVA test was performed to compare significant differences in attention and memory scores among the three groups. Table 2 shows that the TCG had the highest mean scores in most of the attention and memory subtests. The differences were found to be statistically significant in sustained and alternating attention as reflected by the CTT 1 and 2 . Memory scores, as measured by the RMBT (total standard and screening scores) and HKLLT (acquisition, semantic clustering, subjective organization-block condition; acquisition and retrieval-random condition), respectively, showed selective differences among the three groups (Table 2).

The post hoc tests of ANOVA also showed that the TCG had statistically significant better performance in 5 of the 12 HKLLT subtests than that of the EG and CG.). Table 3 shows one of five positive results (as shown in Table 2) obtained from the HKLLT, namely, Memory acquisition-blocked condition (i.e., information was presented on three consecutive trials before subjects were asked to recall as much of the given information as possible). It was found that $t^{\prime} a i$ chi performed better in information acquisition (the encoding) that the two other groups.

\section{Discussion}

In this study, we examined the relationship between cognitive functions (attention and memory) and $t^{\prime} a i$ chi practitioners, EG and CG similar in age, gender, and education level in Hong Kong Chinese older adults. We found that in comparison with the performance of an EG and a CG, the TCG did better in attention (sustained and divided attention) and memory tests (everyday memory function and encoding, recall/organization of verbal information) (Table 2). These findings provided more objective evidence to support the suggestion by previous investigator that $t^{\prime} a i$ chi could provide the mental training (heightened attention, monitoring movement from memory, and self-initiated action) and prerequisites such as anxiety reduction and relief from depression, ${ }^{20}$ which may lead to better attention to stimuli and information encoding and retrieval. ${ }^{18,33}$

Table 2. Analysis of Variance on Attention and Memory Test Scores among T'ai Chi (TCG), Exercise (EG), AND CONTROL Groups (CG)

\begin{tabular}{|c|c|c|c|c|}
\hline Tests (score range) & Mean TCG, EG, CG & Adjusted mean TCG, EG, CG & $\mathrm{F}$ & $\mathrm{p}$ \\
\hline \multicolumn{5}{|l|}{ Color Trails $1^{\mathrm{a}}$} \\
\hline Sustained attention (sec) & $36.73,36.37,62.59$ & $69.09,80.65,90.35$ & 2.69 & $0.03^{*}$ \\
\hline \multicolumn{5}{|l|}{ Color Trails $2^{\mathrm{a}}$} \\
\hline Divided attention (sec) & $63.67,88.86,141.5$ & $143.57,169.30,198.81$ & 1.63 & $0.02^{*}$ \\
\hline \multicolumn{5}{|l|}{ RBMT } \\
\hline Total standard score (0-24) & $3.56,3.55,3.74$ & $19.07,17.67,17.15$ & 3.49 & $0.03^{*}$ \\
\hline Total screening score $(0-12)$ & $2.32,1.96,2.29$ & $8.56,7.61,7.54$ & 3.19 & $0.04^{*}$ \\
\hline \multicolumn{5}{|l|}{ HKLLT learning trial (trials $1+2+3$ ) } \\
\hline Random (0-48) & $7.05,7.45,7.58$ & $28.13,25.31,23.60$ & 5.43 & $0.005^{* * *}$ \\
\hline Blocked $(0-48)$ & $8.34,8.37,10.40$ & $33.28,28.52,25.68$ & 9.41 & $0.00^{* * *}$ \\
\hline \multicolumn{5}{|l|}{$\begin{array}{l}\text { HKLLT delayed recall }(0-16)<B R />\text { (Trial } 4-5 \text { for } \\
\quad \text { random condition; trial } 5-7 \text { for blocked conditions) }\end{array}$} \\
\hline Short delay-random & $1.66,2.0,1.84$ & $1.16,0.65,0.64$ & 1.1 & 0.34 \\
\hline Long delay-random & $1.85,1.85,1.99$ & $1.07,0.46,0.7$ & 1.22 & 0.30 \\
\hline Short delay-blocked & $1.68,2.15,2.33$ & $1.13,0.4,0.16$ & 2.49 & 0.09 \\
\hline Long delay-blocked & $1.64,2.36,2.09$ & $1.15,0.36,0.58$ & 1.75 & 0.18 \\
\hline \multicolumn{5}{|l|}{$\begin{array}{l}\text { HKLLT retrieval (0-16): Difference between 30-min } \\
\text { delay and recognition task }\end{array}$} \\
\hline Random & $2.83,2.38,2.65$ & $-3.37,-4.27,-4.02$ & 1.46 & 0.24 \\
\hline Blocked & $2.22,2.7,2.68$ & $-1.91,-2.83,-3.37$ & 4.12 & $0.018^{*}$ \\
\hline \multicolumn{5}{|l|}{ HKLLT semantic clustering score $(0-12)$} \\
\hline Random (in trial 3) & $3.04,3.04,2.46$ & $4.33,3.74,3.34$ & 1.56 & 0.22 \\
\hline \multicolumn{5}{|l|}{ HKLLT Subjective organization $(0-4)$} \\
\hline $\begin{array}{l}\text { Random (average of first score - trial } 1 \text { and } 2 \\
\text { and second score - trials } 2 \text { and 3) }\end{array}$ & $1.23,1.69,1.35$ & $1.82,1.81,1.47$ & 0.97 & 0.38 \\
\hline \multicolumn{5}{|l|}{ HKLLT semantic clustering score $(0-12)$} \\
\hline Blocked (in trial 3) & $2.78,3.12,3.17$ & $9.15,7.29,6.29$ & 11.5 & $0.00^{* * *}$ \\
\hline $\begin{array}{l}\text { HKLLT subjective organization }(0-4)-\text { blocked } \\
\text { (average of first score - trials } 1 \text { and } 2 \text { and second } \\
\text { score - trials } 2 \text { and } 3 \text { ) }\end{array}$ & $2.27,2.16,1.97$ & $4.19,3.24,2.56$ & 8.16 & $0.00^{* * *}$ \\
\hline
\end{tabular}

${ }^{*} p<0.05,{ }^{* * *} p<0.001$

a Reverse-coded measure for Color Trails tests.

RBMT, Rivermead Behavioral Memory Test; HKLLT, Hong Kong List Learning Test. 
Table 3. Post-hoc Multiple Comparisons of Memory Acquisition Function-Blocked Condition of the Hong Kong List Learning Test by Tukey Honestly Significant Difference (HSD) Test Among $T^{\prime}$ AI $C$ Hi (TCG), Exercise (EG), AND Control Groups (CG)

\begin{tabular}{|c|c|c|c|c|c|c|}
\hline \multicolumn{2}{|c|}{ Group } & \multirow[b]{2}{*}{ Mean difference (I-J) } & \multirow[b]{2}{*}{ Standard error } & \multirow[b]{2}{*}{$\mathrm{p}$ value } & \multicolumn{2}{|c|}{$95 \%$ Confidence interval } \\
\hline (I) Subject group & (J) Subject group & & & & Lower bound & Upper bound \\
\hline \multirow[t]{2}{*}{ T'ai chi } & Exercise & 5.4626 & 1.90726 & $0.013^{*}$ & 0.9415 & 9.9836 \\
\hline & Control & 7.5693 & 1.95664 & $0.000^{* * *}$ & 2.9312 & 12.2074 \\
\hline \multirow[t]{2}{*}{ Exercise } & T'ai chi & -5.4626 & 1.90726 & $0.013^{*}$ & -9.9836 & -0.9415 \\
\hline & Control & 2.1067 & 1.88378 & 0.505 & -2.3587 & 6.5721 \\
\hline \multirow[t]{2}{*}{ Control } & $T^{\prime} a i$ chi & -7.5693 & 1.95664 & $0.000^{* * *}$ & -12.2074 & -2.9312 \\
\hline & Exercise & -2.1067 & 1.88378 & 0.505 & -6.5721 & 2.3587 \\
\hline
\end{tabular}

${ }^{*} p<0.05 ; * * *<0.01$.

Tukey HSD: one of the commonly used post-hoc tests for further analyzing analysis of variance data.

TCG performed better than EG and CG in information acquisition.

No significant difference between exercise and control group in information acquisition.

Both the TCG and the EG performed better than the CG, but the TCG performed even better than the EG in many cognitive areas, such as in the acquisition subtest (Tables 2 and 3). The finding that both TCG and EG performed better than the CG supported previous findings that exercises are cognition enhancing ${ }^{9}$ among older adults, but $t^{\prime}$ ai chi practitioners demonstrated even better cognitive performance than the EG. This seems to be despite the length of practice being longer in the EG (11 years) than in the TCG ( 7.8 years). Again, the possible explanation would be due to an additional effect of $t^{\prime} a i$ chi, as a body-mind exercise, in augmenting both cognitive stimulation and alleviation of anxiety, apart from exercise-only benefits such as increase blood flow in the brain.

The present study clearly had several limitations. It cannot quantify the causal effects of $t^{\prime}$ ai chi on older adults' cognitive function, due to limitations of research design, participant sample size, types of measurement, and statistical analysis. CTT assesses both motor and attentional component and may not be the best way to assess attention. One might expect that $t^{\prime}$ ai chi practice would benefit the motor component of this test as much or more than the attentional component. Moreover, there can be other possible explanations, in addition to the effects of $t^{\prime} a i$ chi on memory and attention. It may be that this group of people chose to do $t^{\prime} a i$ chi because they have good memory and attention, and thus are able to do it well. It is also possible that advocating the need to memorize more complicated t'ai chi movements may deter people who could benefit from some basic activity.

Despite such possibilities, it is evident that the possible use of mind-body exercise ( $t^{\prime} a i$ chi) in services may cater for mind-enhancing needs of older adults who are facing normal or pathological aging. The challenge of $t^{\prime} a i$ chi service implementation may bring about mind-set, behavioral change in older adults, practitioners, and service providers in the long run. In addition, this study serves the purpose of exploratory function and can be viewed as forming a foundation for large scale randomized controlled trial in future. Past researchers have suggested that the cognitive benefits stem from increased blood flow; the current work provides a potentially different explanation for cognitive effects. This may include additional emotional-loaded factors that are supposed to work hand-in-hand with cognitive factors, and possibly can improve self-efficacy or motivation when performing t'ai chi.

\section{Conclusions}

Our findings in this cross-sectional study provides initial evidence that $t^{\prime} a i$ chi practitioners had significantly better attention and memory functions than the exercise group and health control group similar in age, gender, educational and emotional level. Older adults should be encouraged to take up regular exercise, and mind-body exercise might have additional benefits toward cognitive functions and may delay decline due to normal or pathological aging such as dementia. We were unable to examine possible causal relationships, and there is a need for a prospective study to be conducted with a randomized, controlled trial design. Future study along this line is recommended. Ways to standardize the different forms of $t^{\prime} a i$ chi should be explored for basic neuroscience research and to support $t^{\prime}$ ai chi in promoting an increase in cognitive functions. Future study comparing $t^{\prime} a i$ chi performance to a single form of different exercise is also recommended.

\section{Acknowledgments}

This project was funded by the Area of Strategic Development Fund: Centre for East-Meets-West in Rehabilitation Sciences awarded to Christina Hui-Chan, David Man, and William Tsang by The Hong Kong Polytechnic University, Hong Kong.

\section{Disclosure Statement}

No competing financial interests exist.

\section{References}

1. Aniansson A, Grimby G, Rundgren A, et al. Physical training in old men. Age Ageing 1980;9:186-187.

2. Pyka G, Lindenberger E, Charette S, Marcus R. Muscle strength and fiber adaptations to a year-long resistance training program in elderly men and women. J Gerontol 1994;49:22-27.

3. Sandlund ES, Norlander T. The effects of t'ai chi chuan relaxation and exercise on stress responses and well-being: An overview of research. Int J Stress Manage 2000;7:139-149. 
4. Dustman RE, Enunerson RY, Ruhling RO, et al. Age and fitness effects on EEG, ERPs, visual sensitivity, and cognition. Neurobiol Aging 1990;11:193-200.

5. DeCarli C. Mild cognitive impairment: Prevalence, prognosis, aetiology, and treatment. Lancet Neurol 2003;2:15-21.

6. Lopez OL, Kuller LH, Becker JT, et al. Incidence of dementia in mild cognitive impairment in the cardiovascular health study cognition study. Arch Neurol 2007;64:416-420.

7. Hototian SR, Lopes MA, Azevedo D, et al. Prevalence of cognitive and functional impairment in a community sample from São Paulo, Brazil. Dement Geriatr Cogn Disord 2008;25:135-143.

8. Lam LCW, Tam CWC, Lui VWC, et al. Prevalence of very mild and mild dementia in community dwelling older Chinese people in Hong Kong. Int Psychoger 2008;20:135-148.

9. Colcombe S, Kramer AF. Fitness effects on the cognitive function of older adults: A meta-analytic study. Psychol Sci 2003;14:125-130.

10. MacPherson SE, Phillips LH, Della SS. Age, executive function, and social decision making: A dorsolateral prefrontal theory of cognitive aging. Psychol Aging 2002;17:598-609.

11. Wahbeh H, Oken BS. Mind-body interventions: Application in neurology. Neurology 2008;70:2321-2328.

12. Kuramoto A. Therapeutic effects of t'ai chi exercise: Research review. Wisc Med J 2006;105:42-46.

13. Verhagen AP, Immink $M$, van der Meulen A, BiermaZeinstra S. The efficacy of $t^{\prime}$ ai chi chuan in older adults: A systematic review. Fam Pract 2004;21:107-113.

14. Hogan M. Physical and cognitive activity and exercises for older adults: A review. Int J Aging Hum Dev 2005;60:95-126.

15. Wayne PM, Kaptchuck TJ. Challenges inherent to t'ai chi research: Part I. T'ai chi as a complex multi-component intervention. J Altern Complement Med 2008;14:95-102.

16. Chan SP, Luk TC, Hong Y. Kinematic and electromyographic analysis of the push movement of tai chi. Br J Sports Med 2003;37:339-344.

17. Melzer I, Oddsson LI. The effect of a cognitive task on voluntary step execution in healthy elderly and young individuals. J Am Geriatr Soc 2004;52:1255-1262.

18. Banich MT. Cognitive Neuroscience and Neuropsychology. New York, Boston: Houghton Mifflin, 2004.

19. Friedman R, Myers P, Sobel D. Behavioral medicine, clinical health psychology and cost offset. Health Psychol 1995;14: 509-518.

20. Ralph LF. Mind-body fitness: Encouraging prospects for primary and secondary prevention. J Cardiovas Nurs 1997; 11:53-65.
21. Yau KSM, Packer IT. Health and well-being through Tai-Chi: Perceptions of older adults in Hong Kong. Leisure Study 2002;21:163-178.

22. Shah $S$, Cooper B. Issues in the choice of activities of daily living assessment. Aus Occ Ther J 1993;40:77-82.

23. Shah S, Vanclay F, Cooper B. Improving the sensitivity of the Barthel Index for stroke rehabilitation. J Clin Epidemiol 1989;4:703-709.

24. Sheikh JI, Yesavage JA. Geriatric Depression Scale: Recent evidence and development of a shorter version. Clin Gerontol 1986;5:165-172.

25. Yesavage JA, Brink TL, Rose TL, et al. Development and validation of a geriatric depression rating scale: A preliminary report. Psychol Res 1983;17:27.

26. Chiu H, Lee HC, Chung WS, Kwong PK. Reliability and validity of the Cantonese version of mini-mental state examination: A preliminary study. HK J Psychiatry 1994;4:425-428.

27. Lee HCR, Chiu HFK, Kwok PK. Cross validation of the Geriatric Depression Scale Form in the Hong Kong elderly. B HK Psychol Soc 1994;32/33:72-73.

28. D'Elia LFD, Satz P, Uchiyama CL, White T. Color Trails: Professional Manual. Olessa, FL: Psychological Assessment Resources, 1996.

29. Man DWK, Li R. Assessing Chinese older adults' memory abilities: Validation of the Chinese version of the Rivermead Memory Test. Clin Gerontol 2001;24:27-36.

30. Neuro-Rehabilitation Working Group. Validation of Rivermead Behavioral Memory Test-Cantonese version. HK J Occ Ther 1996;9:18-24.

31. Wilson BA, Cockburn J, Baddeley AD. The Rivermead Behavioral Memory Battery. Titchfield, Fareham, Hants: Thames Valley Test Company, 1989.

32. Chan AS, Kwok LC. Hong Kong List Learning Test. Hong Kong: The Chinese University of Hong Kong, 1999.

33. Cook AS, Woollacott MH. Motor Control Theory and Practical Applications. Baltimore, MD: Lippincott Williams and Wilkins, 2001.

Address correspondence to: David W.K. Man, PhD

Department of Rehabilitation Services The Hong Kong Polytechnic University Cheong Wan Road Hung Hom, Kowloon Hong Kong

E-mail: David.Man@polyu.edu.hk 
Copyright of Journal of Alternative \& Complementary Medicine is the property of Mary Ann Liebert, Inc. and its content may not be copied or emailed to multiple sites or posted to a listserv without the copyright holder's express written permission. However, users may print, download, or email articles for individual use. 